\title{
Surgeon and staff radiation exposure in minimally invasive spinal surgery: prospective series using a personal dosimeter
}

\author{
Jakub Godzik, MD, ${ }^{1}$ George M. Mastorakos, BS, ${ }^{2}$ Gautam Nayar, BS, ${ }^{3}$ William D. Hunter, MD, ${ }^{4}$ and \\ Luis M. Tumialán, MD1,5 \\ ${ }^{1}$ Department of Neurosurgery, Barrow Neurological Institute, St. Joseph's Hospital and Medical Center, Phoenix, Arizona; ${ }^{2}$ Mayo \\ Clinic Alix School of Medicine, Scottsdale, Arizona; ${ }^{3}$ Duke University School of Medicine, Department of Neurosurgery, Durham, \\ North Carolina; ${ }^{4}$ Department of Neurosurgery, CaroMont Health Regional Medical Center, Gastonia, North Carolina; and ${ }^{5}$ Spine \\ Group Arizona, HonorHealth, Greenbaum Surgical Specialty Hospital, Scottsdale, Arizona
}

OBJECTIVE The level of radiation awareness by surgeons and residents in spinal surgery does not match the ubiquity of fluoroscopy in operating rooms in the United States. The present method of monitoring radiation exposure may contribute to the current deficiency in radiation awareness. Current dosimeters involve a considerable lag from the time that the surgical team is exposed to radiation to the time that they are provided with that exposure data. The objective of the current study was to assess the feasibility of monitoring radiation exposure in operating room personnel during lateral transpsoas lumbar interbody fusion (LLIF) and minimally invasive transforaminal lumbar interbody fusion (MI-TLIF) procedures by using a wearable personal device with real-time feedback.

METHODS Operating room staff participating in minimally invasive surgical procedures under a single surgeon during a 6 -month period were prospectively enrolled in this study. All radiation dose exposures were recorded for each member of the surgical team (surgeon, assistant surgeon, scrub nurse, and circulating nurse) using a personal dosimeter (DoseAware). Radiation doses were recorded in microsieverts ( $\mu S v)$. Comparisons between groups were made using ANOVA with the Tukey post hoc test and Student t-test.

RESULTS Thirty-nine patients underwent interbody fusions: 25 underwent LLIF procedures (14 LLIF alone, 11 LLIF with percutaneous screw placement [PSP]) and 14 underwent MI-TLIF. For each operative scenario per spinal level, the surgeon experienced significantly higher $(p<0.035)$ average radiation exposure (LLIF: $167.9 \mu S v$, LLIF+PSP: $424.2 \mu S v$, MI-TLIF: $397.9 \mu \mathrm{Sv}$ ) than other members of the team, followed by the assistant surgeon (LLIF: $149.7 \mu$ Sv, LLIF+PSP: $242.3 \mu$ Sv, MI-TLIF: $274.9 \mu S v)$. The scrub nurse (LLIF: $15.4 \mu S v$, LLIF+PSP: $125.7 \mu S v$, MI-TLIF: $183.0 \mu S v$ ) and circulating nurse (LLIF: $1.2 \mu \mathrm{Sv}$, LLIF+PSP: $9.2 \mu \mathrm{Sv}$, MI-TLIF: $102.3 \mu \mathrm{Sv}$ ) experienced significantly lower exposures. Radiation exposure was not correlated with the patient's body mass index ( $p \geq 0.233$ ); however, it was positively correlated with increasing patient age ( $p \leq 0.004)$.

CONCLUSIONS Real-time monitoring of radiation exposure is currently feasible and shortens the time between exposure and the availability of information regarding that exposure. A shortened feedback loop that offers more reliable and immediate data would conceivably raise the level of concern for radiation exposure in spinal surgeries and could alter patterns of behavior, leading to decreased exposures. Further studies are ongoing to determine the effect of real-time dosimetry in spinal surgery.

https://thejns.org/doi/abs/10.3171/2019.11.SPINE19448

KEYWORDS lateral lumbar interbody fusion; minimally invasive surgery; percutaneous pedicle screws; radiation exposure; radiation safety; surgical technique

ABBREVIATIONS ALARA = as low as is reasonably achievable; $\mathrm{BMI}=$ body mass index; LLIF = lateral lumbar interbody fusion; $\mathrm{Ml}$-TLIF = minimally invasive transforaminal lumbar interbody fusion; PSP = percutaneous screw placement.

SUBMITTED April 12, 2019. ACCEPTED November 25, 2019

INCLUDE WHEN CITING Published online February 7, 2020; DOI: 10.3171/2019.11.SPINE19448. 
$\mathrm{I}$ ONIZING radiation is the unavoidable consequence of visualizing the anatomy for any spinal procedure. Regardless of the spinal procedure being performed, cross-table lateral radiography, fluoroscopy, and computer-assisted navigation all require radiation to generate an image. The rise of minimally invasive procedures has resulted in even greater reliance on imaging given the limited fields of exposure and constrained working channels. ${ }^{6}$ The inevitable consequence has been higher exposures to radiation for a given procedure. ${ }^{17}$

Studies have consistently demonstrated higher radiation exposures in minimally invasive procedures than in open procedures. ${ }^{17}$ Percutaneous instrumentation of the spine is completely dependent on some form of imaging, whether from fluoroscopy or computer-assisted navigation. Minimally invasive transforaminal lumbar interbody fusion (MI-TLIF), in particular, represents a notable source of surgical staff radiation exposure. In addition, the transpsoas approach to the lumbar spine, which has been introduced in the past decade, has brought the surgeon inextricably closer to the source of radiation for a surgery that is highly dependent on imaging for the entire procedure. And although exposures to radiation have been increasing, there has not been a commensurate increase in radiation awareness and education. A potential cause of this divide may be the disconnect between exposure and knowledge about the amount of exposure per procedure. At the heart of that issue is dosimetry.

Commonly used dosimeters are assigned to healthcare professionals who are exposed to radiation, and these dosimeters are collected monthly or quarterly. ${ }^{5}$ Infrequent radiation verification means that there is considerable lag time between performing a series of cases and obtaining information about the amount of radiation exposure from those cases. This lag time makes focused corrective action challenging. However, if information regarding radiation exposure were collected immediately after a procedure was performed, such feedback would offer the possibility of modifying one's behavior in order to reduce exposure. ${ }^{7}$ In a variety of other specialties and fields, strategic changes in workflow are highly dependent on instantaneous feedback on performance. Applying that same model to radiation exposures in spinal procedures would increase awareness of the radiation exposure that results from a particular case..$^{13}$ That knowledge has the potential to decrease radiation exposures and improve adherence to safety guidelines., ${ }^{911-14}$

In order to explore the hypothesis that real-time feedback on intraoperative radiation exposure will eventually empower the surgical team to make strategic changes in behavior, we wanted to establish the feasibility of using a wearable personal dosimeter with the potential for realtime feedback. The objectives of this study were 1) to prospectively assess the feasibility of obtaining an immediate report on radiation exposure following an operation after using a wearable dosimeter and 2) to provide a quantitative baseline of radiation exposure to operating room staff during routine minimally invasive spinal surgery to illustrate the device's clinical application.

\section{Methods \\ Study Design}

A prospective cohort study was undertaken to compare radiation exposures to operating room personnel during minimally invasive spinal surgery. Personnel included the surgeon, assistant surgeon, circulating nurse, and scrub nurse. The inclusion criteria were as follows: 1) a lumbar surgery performed via the lateral transpsoas lumbar interbody fusion (LLIF) or MI-TLIF approach and 2) involving the intraoperative use of continuous interlaced fluoroscopy for anatomical visualization, 3) in patients at least 18 years of age. All operations were performed by a single surgeon during a 6-month period spanning 2014 and 2015 at a single institution. Collected preoperative variables included the patient's age, sex, and body mass index (BMI), the surgical approach, the number of levels operated on, and the number of screws used. Screw accuracy was not assessed. This study was approved by the institutional review board at St. Joseph's Hospital and Medical Center in Phoenix, Arizona.

\section{Surgical Technique}

Patients underwent either MI-TLIF or LLIF with or without second-stage percutaneous screw placement (PSP). For the MI-TLIF, surgery was performed using two $28-\mathrm{mm}$ incisions at $3.5-4.0 \mathrm{~cm}$ lateral to the midline. After localization was confirmed by 1 or 2 lateral images, pedicle screws were placed in a percutaneous fashion, with attachment of minimally invasive expandable retractors to facilitate unilateral facetectomy, discectomy, and placement of an interbody spacer. For the LLIF (first stage), the patient was placed in the lateral decubitus position, and localization was performed using anteroposterior and lateral fluoroscopy. Surgical access was obtained via a oneincision technique. If necessary, PSP was performed via a second stage with the patient prone.

\section{Imaging and Radiation}

All imaging was performed using a 9-inch OEC 9900 or 9800 C-arm mobile fluoroscopic imaging system (GE Healthcare) with automatic brightness control. Radiation exposures for all members of the surgical team (surgeon, assistant surgeon, circulating nurse, scrub nurse) were collected using DoseAware (Philips Healthcare), a personal dosimeter with proven efficacy in spine and vascular surgery. ${ }^{13}$ All individuals in the operating room wore radiation protection aprons with thyroid collars. The dosimeter was placed on the chest pocket of the lead apron beneath the sterile gown. Radiation exposure was expressed in real time as a cumulative dose in microsieverts $(\mu \mathrm{Sv})$ via the dosimeter. If personnel left for a break, the dosimeter was transferred to their replacement to provide a cumulative dose to the specific staff role rather than the individual. The typical operating room setup is illustrated in Fig. 1.

\section{Statistical Analysis}

Patient demographics were compared between the LLIF, LLIF+PSP, and MI-TLIF groups by univariate analysis. Continuous variables were aggregated as the mean and standard deviation and compared using the Student ttest. Categorical variables were compared using the Fisher exact test after aggregation as proportions. Radiation exposure in LLIF+PSP and MI-TLIF was compared for each 


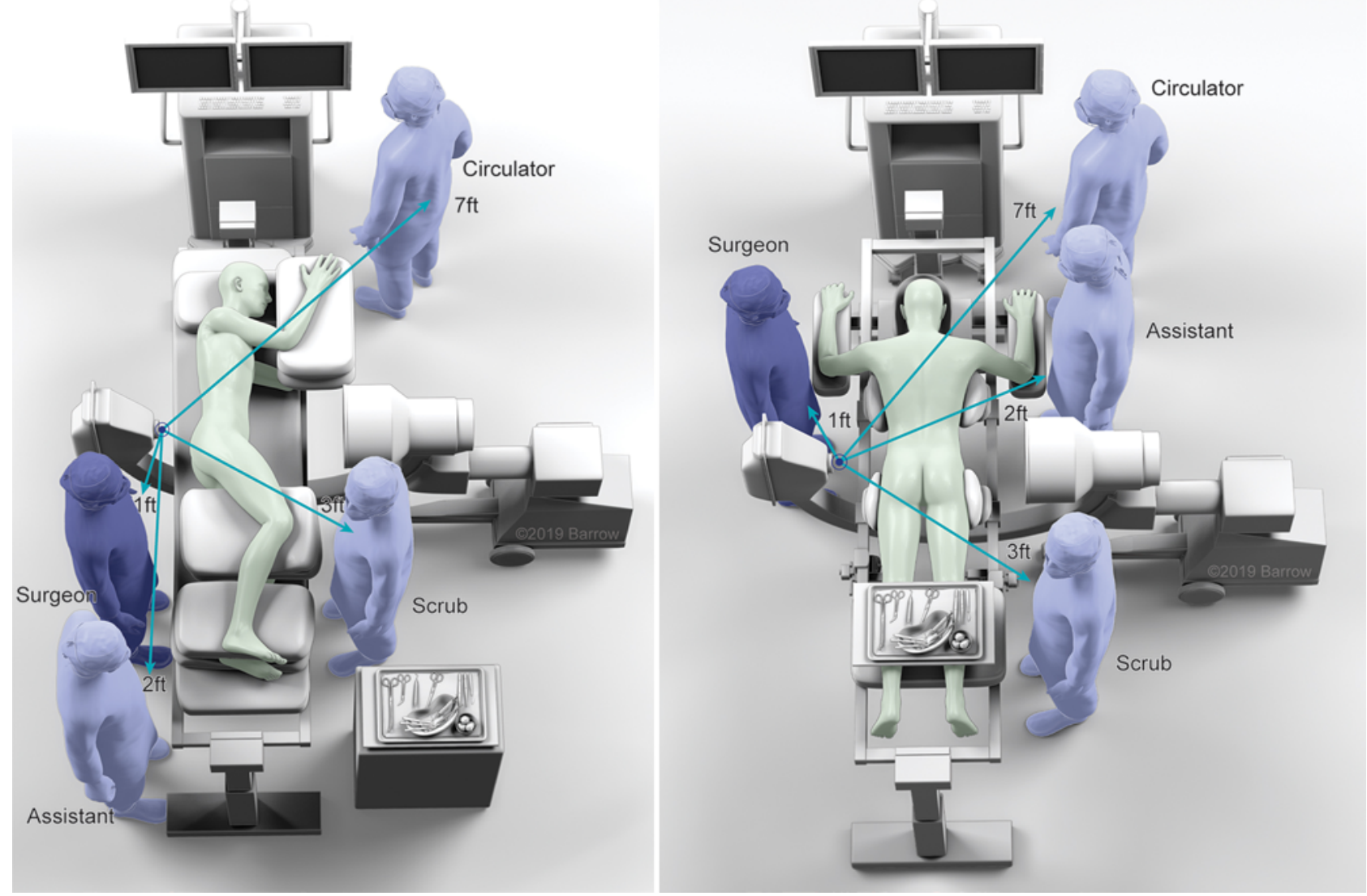

FIG. 1. Artistic renditions of the positions of operating room staff during the LLIF procedure (left) and either a TLIF or a PSP procedure (right). Approximate distances from the radiation source to each staff are indicated. Used with permission from Barrow Neurological Institute, Phoenix, Arizona. Figure is available in color online only.

operating room staff category, as was cumulative exposure for the entire team, using the Student t-test. Radiation exposure between operating room personnel was compared for each approach (LLIF, LLIF+PSP, and MI-TLIF) by ANOVA with the Tukey post hoc test. Finally, per-procedure exposure was calculated for each team member in terms of the number of procedures needed to reach as low as is reasonably achievable (ALARA) level I (125 mrem/ quarter), ALARA level II (375 mrem/quarter), and the maximum occupational radiation exposure threshold of $5000 \mathrm{mrem} / \mathrm{yr}$ to the whole body. ${ }^{5,15,16}$ For the Student ttest, equal or unequal variance was determined using an $\mathrm{F}$ test. Analyses were performed using JMP Pro 13 (SAS Institute). For all tests, significance was set at $p<0.05$.

\section{Results \\ Demographics}

Thirty-nine patients underwent interbody fusions, 25 of whom underwent LLIF procedures (14 LLIF alone and 11 LLIF+PSP) and 14 of whom underwent MI-TLIF. Demographic and surgical data are presented in Table 1. Comparing approaches, there were no significant differences in patient age, sex, or BMI or in the number of levels operated or number of screws placed ( $p \geq 0.179)$. No patients required a repeat procedure to replace or reposition screws.

\section{Operating Room Staff Exposure to Radiation}

Radiation exposures of the operating room staff are presented in Tables 2 and 3 and Fig. 2. The number of procedures needed to exceed the occupational radiation exposure threshold is presented in Table 4. Radiation exposure experienced by the surgeon during all LLIF+PSP procedures (per case and per level) was significantly greater than that experienced by the assistant surgeon $(\mathrm{p}<0.035)$, circulating nurse $(\mathrm{p}<0.0015)$, and scrub nurse $(\mathrm{p}<0.001)$,

TABLE 1. Demographic parameters across procedure type

\begin{tabular}{lcccc}
\hline \multicolumn{1}{c}{ Variable } & LLIF Alone & LLIF+PSP & MI-TLIF & $\begin{array}{c}\mathrm{p} \\
\text { Value }\end{array}$ \\
\hline No. of cases & 14 & 11 & 14 & \\
\hline Patient age in yrs & $58.6 \pm 11.8$ & $55.8 \pm 13.0$ & $65.4 \pm 16.7$ & 0.179 \\
\hline Female sex (\%) & $8(57)$ & $5(45.5)$ & $10(71)$ & 0.409 \\
\hline BMl in kg/m ${ }^{2}$ & $32.8 \pm 6.9$ & $33.7 \pm 6.9$ & $29.9 \pm 3.9$ & 0.252 \\
\hline No. of surgical levels & $1.3 \pm 0.54$ & $1.3 \pm 0.46$ & $1.1 \pm 0.36$ & 0.676 \\
\hline No. of screws & NA $^{*}$ & $4.5 \pm 0.91$ & $4.3 \pm 0.73$ & 0.428 \\
\hline
\end{tabular}

$\mathrm{NA}=$ not applicable.

Data are expressed as the mean \pm standard deviation, unless indicated otherwise.

* No screws were placed for any LLIF-alone procedure. 
TABLE 2. Radiation exposure of operating room staff per case by procedure

\begin{tabular}{lcccc}
\hline & LLIF Alone & LLIF+PSP & MI-TLIF & p Value \\
\hline Surgeon & $208.6 \pm 146.9$ & $541.0 \pm 236.3$ & $408.3 \pm 192.3$ & 0.135 \\
\hline Assistant surgeon & $186.0 \pm 210.0$ & $317.6 \pm 252.0$ & $280.7 \pm 196.8$ & 0.684 \\
\hline Scrub nurse & $19.2 \pm 22.1$ & $155.4 \pm 116.8$ & $185.7 \pm 187.2$ & 0.643 \\
\hline Circulating nurse & $1.6 \pm 1.6$ & $11.3 \pm 11.0$ & $102.4 \pm 164.5$ & 0.059 \\
\hline Total & $413.8 \pm 321.4$ & $1025.3 \pm 494.6$ & $977.1 \pm 580.6$ & 0.828 \\
\hline
\end{tabular}

All data are expressed as the mean \pm standard deviation in $\mu$ Sv.

but it was not significantly greater than that experienced by the assistant surgeon in LLIF and MI-TLIF procedures $(\mathrm{p}>0.25)$. Radiation exposure experienced by the assistant surgeon per case and per level was significantly greater than that experienced by both the circulating nurse and scrub nurse in LLIF ( $p \leq 0.015)$. Exposure for the assistant surgeon was higher than that for the circulating nurse in LLIF+PSP ( $p \leq 0.002)$ but not in MI-TLIF $(p \geq 0.09)$. Exposures for the scrub nurse and circulating nurse were not significantly different for any procedure $(p \geq 0.15)$. Patient $\mathrm{BMI}$ was not correlated with staff radiation exposure $(\mathrm{p} \geq$ 0.233 ), but increasing patient age had a positive correlation with radiation exposure experienced by staff $(\mathrm{p} \leq 0.004)$.

\section{Comparison of Radiation Exposure Between LLIF+PSP and MI-TLIF Procedures}

Overall, there were no significant differences in radiation exposure between MI-TLIF and LLIF+PSP for any team member or for the cumulative team exposure $(\mathrm{p}>$ $0.055)$. Although there was an increase in radiation exposure for the circulating nurse between the LLIF+PSP and MI-TLIF procedures, this difference was not significant $(\mathrm{p}=0.055)$.

\section{Discussion}

When radiation exposure data are available immediately after an event, immediate analysis and action can be taken. Conversely, aggregate data that are collected over a period of time, representing a number of different events, have little value in identifying critical aspects of behavior. In minimally invasive spine surgery, an effort has been made to reduce the amount of ionizing radiation for every procedure. However, the dosimeters commonly used in spine surgery offer data accumulated over a number of cases over the span of weeks, if not months. The consider- able lag time between data acquisition and analysis severely impedes the opportunity to take timely action. To lower radiation, dosimetry data should be available for analysis immediately.

The purpose of this study was to establish the feasibility of real-time dosimetry in spine surgery and to quantify occupational radiation exposure to operating room staff during minimally invasive spinal procedures. Our research demonstrated the utility and ease of using personal radiation monitors with the potential for instantaneous feedback on radiation exposure in the operating room during minimally invasive spinal procedures. Using real-time dosimetry, we observed that the degree of radiation exposure to the operating room team differs substantially between staff members and between different minimally invasive surgical approaches. We noted that the surgeon and assistant surgeon experienced higher levels of radiation exposure than the circulating nurse and scrub nurse across all operations studied, whereas the surgeon experienced significantly greater radiation exposure than the assistant surgeon only in the LLIF+PSP procedures. Furthermore, the surgical team (surgeon, assistant surgeon, scrub nurse, and circulating nurse) experienced similar radiation exposure in MI-TLIF and LLIF+PSP.

Similar exposure rates for the surgeon and assistant surgeon have been reported in studies of radiation exposure in the operating room. In a study of 31 MI-TLIF cases, Funao et al. ${ }^{3}$ demonstrated a 6-, 5-, and 5-mrem effective dose to the surgeon, assistant, and radiology technologist, respectively, using conventional fluoroscopy. These authors reported significantly higher exposure for the surgeon's finger than for any other part of the body (chest, genitals, or lens of the eye); they also found that while the surgeon had higher exposure to the finger than the assistant surgeon, there was no difference between the two staff roles in terms of radiation exposure to the chest. Similarly, Taher

TABLE 3. Radiation exposure of operating room staff per level by procedure

\begin{tabular}{lcccc}
\hline & LLIF Alone & LLIF+PSP & MI-TLIF & p Value \\
\hline Surgeon & $167.9 \pm 125.8$ & $424.2 \pm 119.0$ & $397.9 \pm 207.9$ & 0.713 \\
\hline Assistant surgeon & $149.7 \pm 164.4$ & $242.3 \pm 200.6$ & $274.9 \pm 203.3$ & 0.693 \\
\hline Scrub nurse & $15.4 \pm 21.9$ & $125.7 \pm 96.3$ & $183.0 \pm 189.3$ & 0.338 \\
\hline Circulating nurse & $1.2 \pm 1.2$ & $9.2 \pm 10.8$ & $102.3 \pm 164.6$ & 0.055 \\
\hline Total & $334.2 \pm 278.6$ & $720.5 \pm 357.2$ & $958.1 \pm 607.1$ & 0.407 \\
\hline
\end{tabular}

All data are expressed as the mean \pm standard deviation in $\mu \mathrm{Sv}$. 
Godzik et al.

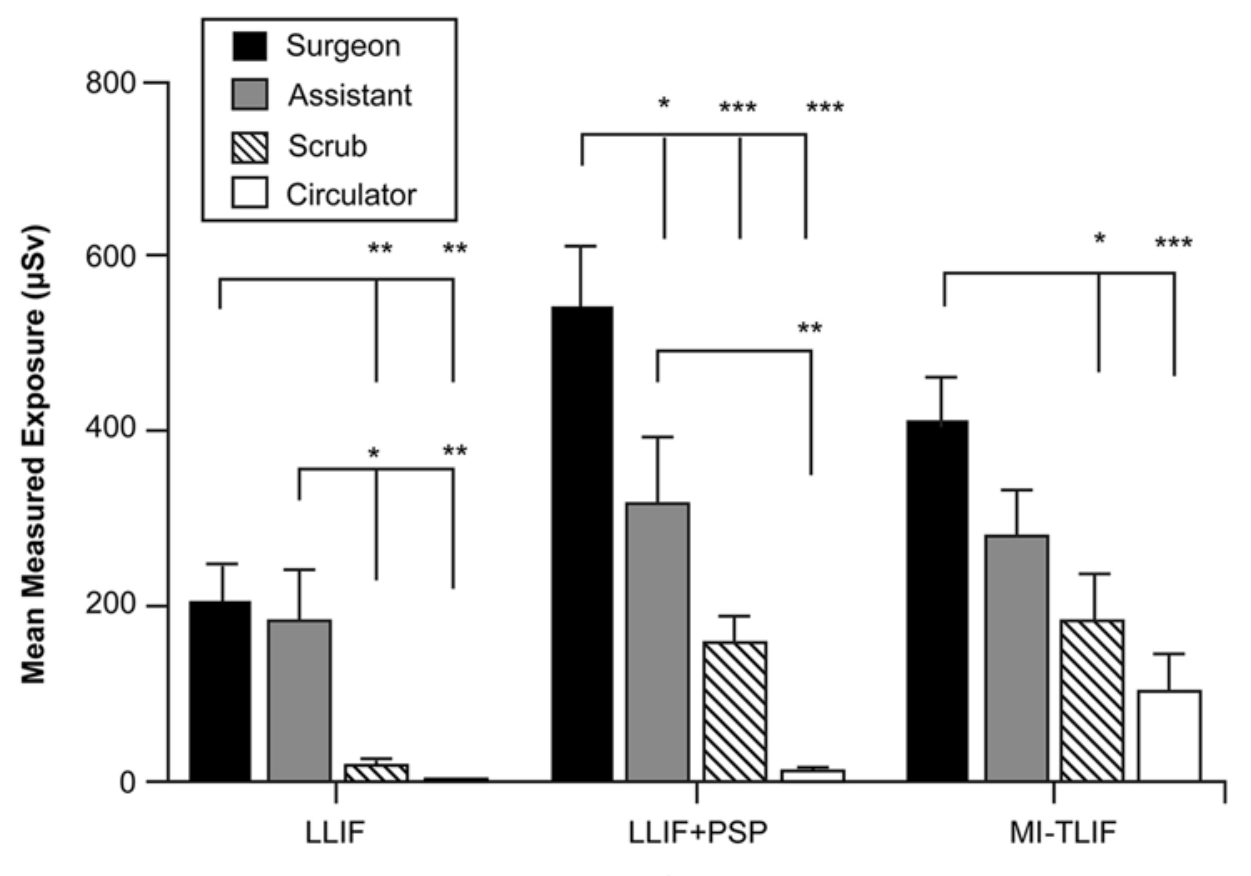

Type of Procedure

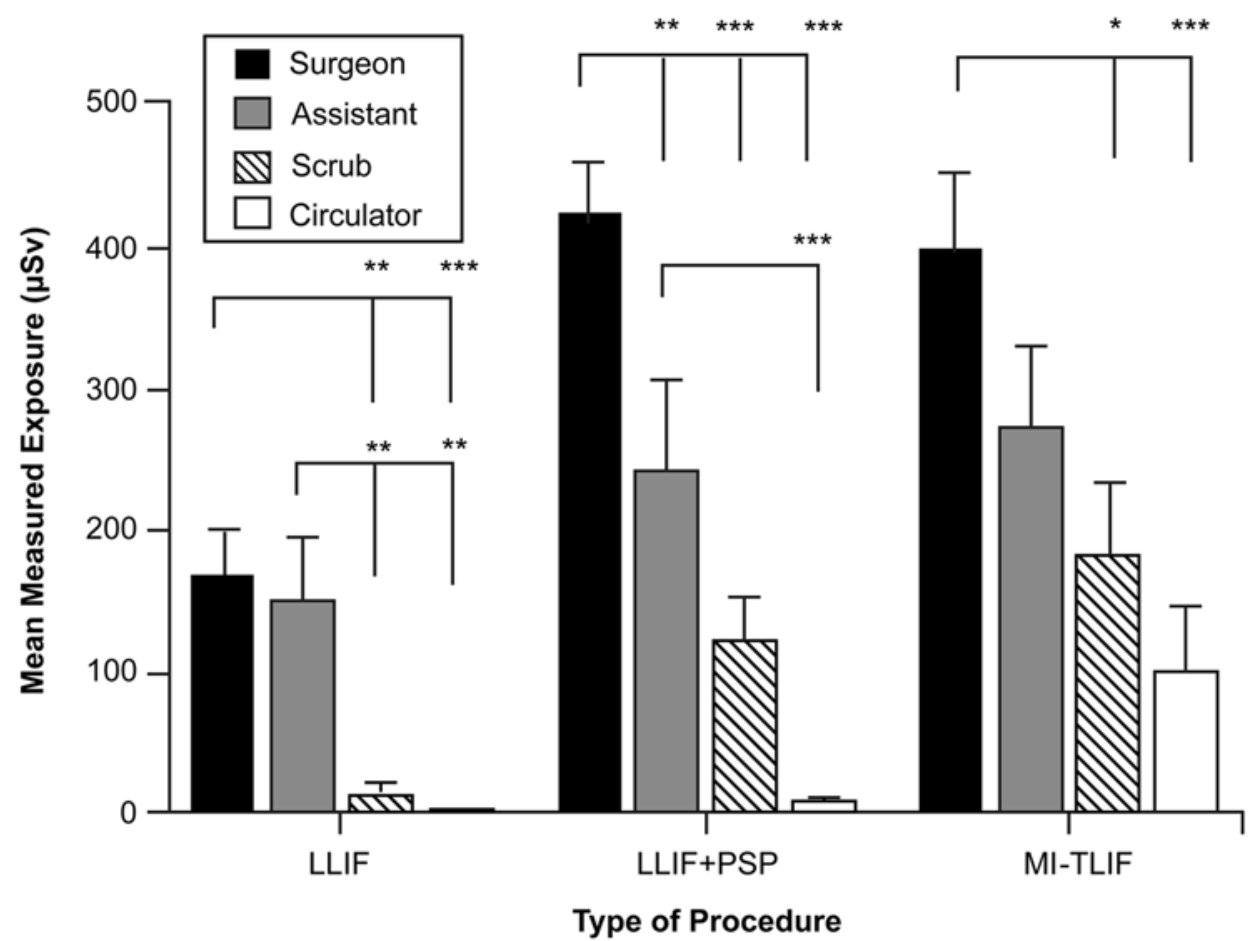

FIG. 2. Radiation exposure of the surgeon, assistant surgeon, scrub nurse, and circulating nurse by surgical approach. Radiation exposure of the personnel was measured using the DoseAware system and organized by surgical approach per case (upper) and per spinal level (lower). ANOVA was performed $(\alpha=0.05)$, and the significance of thresholds is represented as ${ }^{*} p<0.05,{ }^{* *} p<$ 0.01 , and ${ }^{* * *} p<0.001$. Error bars represent standard deviation.

et al. ${ }^{15}$ demonstrated the highest radiation exposure to the ring finger (14.6 mrem), axilla (4.2 mrem), and eye (2.64 mrem) in a study of 18 LLIF surgeries. According to the recommended limit for occupational radiation exposure to the whole body in our study, the surgeon would need to perform between 92 and 239 procedures per year to reach the threshold (Table 4). ${ }^{1,15,16}$ This roughly compares to the magnitude reported by Taher et al..$^{15}$ - between 2703 and 6757 LLIF procedures per year using a threshold of 50,000 $\mathrm{mrem} / \mathrm{yr}$. In the current study, the dosimeter was located at 
TABLE 4. Number of procedures needed to exceed recommended occupational radiation dose limits

\begin{tabular}{lrrr}
\hline \multicolumn{1}{c}{ Dose Limit } & LLIF Alone & LLIF+PSP & MI-TLIF \\
\hline ALARA level I (125 mrem/quarter) & & & \\
\hline$\quad$ Surgeon & 6 & 2 & 3 \\
\hline Assistant surgeon & 7 & 4 & 4 \\
\hline Scrub nurse & 65 & 8 & 7 \\
\hline Circulating nurse & 781 & 111 & 12 \\
\hline ALARA level II (375 mrem/quarter) & & & \\
\hline$\quad$ Surgeon & 18 & 7 & 9 \\
\hline Assistant surgeon & 20 & 12 & 13 \\
\hline Scrub nurse & 195 & 24 & 20 \\
\hline Circulating nurse & 2344 & 332 & 37 \\
\hline Cumulative for the yr, level I (5000 & & & \\
mrem/yr) & & & \\
\hline$\quad$ Surgeon & 239 & 92 & 112 \\
\hline$\quad$ Assistant surgeon & 268 & 157 & 178 \\
\hline$\quad$ Scrub nurse & 2604 & 322 & 269 \\
\hline$\quad$ Circulating nurse & 31250 & 4424 & 488 \\
\hline
\end{tabular}

the chest level in an effort to replicate the commonly worn location of radiation badges. The location of the dosimeter may explain why more differences were not seen between the surgeon and assistant, with significant differences in radiation exposure observed only in the LLIF+PSP procedures.

Studies have shown that distance is important in reducing scattered radiation risk. In a simulated operative environment, Nelson et al..$^{10}$ described a strong relationship between proximity and radiation exposure, with significantly higher doses to the surgeon (0.047-0.546 mR) compared to doses to the assistant $(0.016-0.053 \mathrm{mR})$, anesthesiologist (0.04-0.17 mR), and radiology technologist (0.002-0.008 $\mathrm{mR})$. Although several studies have described exposure during endovascular procedures, few have documented exposure rates of the circulating nurse and scrub nurse. Mohapatra et al. ${ }^{8}$ reported the highest radiation exposure to the anesthesiologist, with the lowest doses for the scrub nurse and radiation technologist. They also found that the operator dose was correlated to patient weight. Similar to Mohapatra, we found remarkable variability in radiation across different personnel, but there was also variability within the same staff position category. Such variability in exposures suggests that not only is distance from radiation emission critical, but so is behavior. Some assistants may move away from the field, while others may stand close. As exposure is dictated by distance from the source, behaviors likely altered the ultimate doses, further emphasizing the potential for real-time feedback in modifying intraoperative behavior and ultimately an individual's cumulative dose.

The circulating nurse and scrub nurse are also critical members of the surgical team. Few studies have explored the radiation exposure of these team members, even though a review of national occupational information showed that these roles are predominantly filled by women of childbearing age. Although the scrub nurse and circulating nurse have lower exposure than the surgeon and assistant surgeon, they are nonetheless exposed to an amount of radiation greater than zero. Given the risks of ionizing radiation exposure on female fertility, ${ }^{1,2,4}$ individuals in these occupations should be further educated on the risks of radiation exposure and the importance of radiation safety (https://datausa.io/profile/cip/surgicaltechnologist).

To keep radiation exposure to patients and staff as low as reasonably achievable, compliance with radiation protection principles is crucial. Our data establish baseline exposure rates and may provide a foundation for further reduction of exposure by allowing operating room personnel to modify their intraoperative strategies. Previous authors have demonstrated the utility of real-time feedback for cardiac, pediatric, orthopedic, and pain intervention procedures. ${ }^{9}, 11-14$ Müller et al. compared personnel wearing DoseAware dosimeter badges with retrospectively matched historical cohorts and showed reduced rates of exposure among the orthopedic surgeons with real-time feedback. ${ }^{9}$ Slegers et al. ${ }^{14}$ reported on the impact of real-time radiation dose feedback plus coaching on the reduction of scatter radiation dose on pain physicians during interventional pain procedures, citing a radiation reduction of $46.6 \%$ across 330 procedures. Although we did not measure the impact of coaching, their study demonstrates the potential of integrating real-time data in a clinical setting to strengthen intraoperative strategies to minimize radiation exposure in the future.

\section{Study Limitations}

Several key limitations may limit the generalizability of our study. Although this study was prospective, the participants were not blinded to the real-time dosimetry readings, potentially leading to altered behavior and subjecting the study to bias. In addition, the impact of realtime dosimetry on intraoperative behavior was not assessed, a comparison with conventional dosimetry was not performed, and staff personnel were not interviewed regarding comfort or usability of the device, thus limiting our ability to assess behavioral changes. Furthermore, we did not record the number of radiographs taken, fluoroscopy time, fluoroscope settings from case to case, length of surgical procedure, staff distance from the fluoroscope, or screw accuracy, thereby limiting our ability to analyze the influence of specific case characteristics on the radiation exposure of operating room personnel. However, these surgical cases were performed within a short period of time, reflecting few changes in technique and limiting the effects of the surgeon's learning curve. In addition, given logistical difficulties, we did not compare the wearable dosimeter with a gold-standard device, such as the thermoluminescent dosimeter, which limits our ability to comment on the device's efficacy in detecting radiation exposure. Finally, our study was limited to the staff categories selected because of the limited number of available dosimeters. Thus, while it is equally crucial to consider the impact of radiation exposure on the anesthesiologist and radiation technologist, our study was focused only on the surgeon, assistant surgeon, circulating nurse, and scrub nurse. 


\section{Conclusions}

Real-time dosimetry in spine surgery is feasible and may enable surgical staff to maximize intraoperative strategies, barriers, and positioning to minimize radiation exposure, although such minimization strategies were not evaluated in the current study. We propose future studies to investigate the ability of real-time feedback to provide increased awareness of scatter radiation and strategic insight into surgeon and staff positioning.

\section{Acknowledgments}

We thank the staff of Neuroscience Publications at Barrow Neurological Institute for assistance with manuscript preparation.

\section{References}

1. Figà-Talamanca I: Occupational risk factors and reproductive health of women. Occup Med (Lond) 56:521-531, 2006

2. Figà-Talamanca I: Reproductive problems among women health care workers: epidemiologic evidence and preventive strategies. Epidemiol Rev 22:249-260, 2000

3. Funao H, Ishii K, Momoshima S, Iwanami A, Hosogane N, Watanabe K, et al: Surgeons' exposure to radiation in singleand multi-level minimally invasive transforaminal lumbar interbody fusion; a prospective study. PLoS One 9:e95233, 2014

4. Goldberg MS, Mayo NE, Levy AR, Scott SC, Poîtras B: Adverse reproductive outcomes among women exposed to low levels of ionizing radiation from diagnostic radiography for adolescent idiopathic scoliosis. Epidemiology 9:271-278, 1998

5. Interagency Working Group on Medical Radiation: Federal Guidance Report No. 14. Radiation Protection Guidance for Diagnostic and Interventional X-Ray Procedures. Washington, DC: U.S. Environmental Protection Agency, 2014 (https://www.epa.gov/radiation/federal-guidance-reportno-14-radiation-protection-guidance-diagnostic-and-interventional) [Accessed December 6, 2019]

6. Khan NR, Clark AJ, Lee SL, Venable GT, Rossi NB, Foley $\mathrm{KT}$ : Surgical outcomes for minimally invasive vs open transforaminal lumbar interbody fusion: an updated systematic review and meta-analysis. Neurosurgery 77:847-874, 2015

7. Lintmeijer LL, van Soest AJ, Robbers FS, Hofmijster MJ, Beek PJ: Real-time feedback on mechanical power output: facilitating crew rowers' compliance with prescribed training intensity. Int J Sports Physiol Perform 14:303-309, 2018

8. Mohapatra A, Greenberg RK, Mastracci TM, Eagleton MJ, Thornsberry B: Radiation exposure to operating room personnel and patients during endovascular procedures. J Vasc Surg 58:702-709, 2013

9. Müller MC, Welle K, Strauss A, Naehle PC, Pennekamp PH, Weber O, et al: Real-time dosimetry reduces radiation exposure of orthopaedic surgeons. Orthop Traumatol Surg Res 100:947-951, 2014
10. Nelson EM, Monazzam SM, Kim KD, Seibert JA, Klineberg EO: Intraoperative fluoroscopy, portable X-ray, and CT: patient and operating room personnel radiation exposure in spinal surgery. Spine J 14:2985-2991, 2014

11. Ordiales JM, Nogales JM, Sánchez-Casanueva R, Vano E, Fernández JM, Alvarez FJ, et al: Reduction of occupational radiation dose in staff at the cardiac catheterisation laboratory by protective material placed on the patient. Radiat Prot Dosimetry 165:272-275, 2015

12. Racadio J, Nachabe R, Carelsen B, Racadio J, Hilvert N, Johnson N, et al: Effect of real-time radiation dose feedback on pediatric interventional radiology staff radiation exposure. J Vasc Interv Radiol 25:119-126, 2014

13. Sanchez R, Vano E, Fernandez JM, Gallego JJ: Staff radiation doses in a real-time display inside the angiography room. Cardiovasc Intervent Radiol 33:1210-1214, 2010

14. Slegers AS, Gültuna I, Aukes JA, van Gorp EJ, Blommers FM, Niehof SP, et al: Coaching reduced the radiation dose of pain physicians by half during interventional procedures. Pain Pract 15:400-406, 2015

15. Taher F, Hughes AP, Sama AA, Zeldin R, Schneider R, Holodny EI, et al: 2013 Young Investigator Award winner: How safe is lateral lumbar interbody fusion for the surgeon? A prospective in vivo radiation exposure study. Spine (Phila Pa 1976) 38:1386-1392, 2013

16. Wrixon AD: New ICRP recommendations. J Radiol Prot 28:161-168, 2008

17. Yu E, Khan SN: Does less invasive spine surgery result in increased radiation exposure? A systematic review. Clin Orthop Relat Res 472:1738-1748, 2014

\section{Disclosures}

Dr. Tumialán is a consultant for Medtronic and has ownership in LessRay.

\section{Author Contributions}

Conception and design: Tumialán. Acquisition of data: Nayar, Hunter. Analysis and interpretation of data: Godzik, Mastorakos, Nayar. Drafting the article: Godzik, Mastorakos, Nayar. Critically revising the article: Tumialán. Reviewed submitted version of manuscript: Tumialán, Godzik. Statistical analysis: Mastorakos, Nayar. Administrative/technical/material support: Tumialán, Hunter. Study supervision: Tumialán.

\section{Correspondence}

Luis M. Tumialán: c/o Neuroscience Publications, Barrow Neurological Institute, St. Joseph's Hospital and Medical Center, Phoenix, AZ.neuropub@barrowneuro.org. 